Revue des patrimoines scientifiques et opérationnels

\title{
Relecture de vocabulaires d'architecture : apport de la complexité des représentations numériques dans la caractérisation de formes architecturales
}

Rereading architectural vocabularies: the contribution of the complexity of

digital representations in the characterisation of architectural forms

\section{David Lo Buglio}

\section{(2) OpenEdition}

Journals

Édition électronique

URL : http://journals.openedition.org/insitu/21352

DOI : $10.4000 /$ insitu. 21352

ISSN : $1630-7305$

Éditeur

Ministère de la Culture

Référence électronique

David Lo Buglio, «Relecture de vocabulaires d'architecture : apport de la complexité des

représentations numériques dans la caractérisation de formes architecturales », In Situ [En ligne],

39 | 2019, mis en ligne le 23 mai 2019, consulté le 11 juillet 2019. URL : http://

journals.openedition.org/insitu/21352; DOI : 10.4000/insitu.21352

Ce document a été généré automatiquement le 11 juillet 2019.

\section{c) $(1) \ominus$}

In Situ Revues des patrimoines est mis à disposition selon les termes de la licence Creative Commons Attribution - Pas d'Utilisation Commerciale - Pas de Modification 4.0 International. 


\title{
Relecture de vocabulaires d'architecture : apport de la complexité des représentations numériques dans la caractérisation de formes architecturales
}

\author{
Rereading architectural vocabularies: the contribution of the complexity of \\ digital representations in the characterisation of architectural forms
}

\section{David Lo Buglio}

1 En articulant terminologie, logique fonctionnelle et logique géométrique, la sémantique d'un élément peut être considérée comme un concept d'architecture à part entière. Par sa capacité à structurer et organiser le bâti autour d'un langage commun (affirmation que nous nuancerons par la suite), la sémantique architecturale constitue à bien des égards l'un des fondements des théories d'architecture. En se situant à la croisée de la théorie d'architecture, de l'histoire de l'art et des pratiques du relevé, les vocabulaires d'architecture offrent des tentatives de normalisation sémantique qui pourraient être rediscutées à la lumière des avancées scientifiques récentes dans le domaine de l'étude du bâti.

Depuis de nombreuses années maintenant, les technologies numériques ont durablement investi le champ du relevé et de la valorisation du patrimoine bâti, offrant de nouveaux instruments pour sa conservation et sa médiation. Dans ce contexte, l'application des méthodes de relevé les plus récentes (comme la lasergrammétrie ou la photogrammétrie architecturale) se révèle utile pour l'enregistrement fin d'artefacts architecturaux. Ces nouveaux moyens mis au service du patrimoine favorisent la production de numérisations géométriques complexes capables de restituer l'apparence visuelle du bâti. Néanmoins, l'utilisation de ces moyens à des fins de connaissance reste limitée. Au-delà de l'enjeu épistémologique auquel ce constat conduit, une question doit se poser : ces 
données massives sont-elles susceptibles d'affiner notre compréhension du bâti et de ses évolutions stylistiques dans le temps et dans l'espace?

3 Cet article vise à concilier les enjeux cognitifs propres à l'étude sémantique d'éléments architecturaux et l'exploitation de numérisations complexes. En s'appuyant sur l'étude des similarités morphologiques partagées par un corpus d'éléments architecturaux, l'ambition est de croiser la caractérisation géométrique du corpus (modèle géométrique) avec la description fournie par des vocabulaires d'architecture pour le même type d'élément. Il s'agit d'observer les points de rencontre mais aussi de tension entre une sémantique proposée par l'histoire de l'art et une " sémantique " géométrique issue d'une observation morphologique fine. En mettant en exergue les contradictions entre les vocabulaires eux-mêmes et le modèle géométrique obtenu, cet article discute l'apport des méthodes d'analyse provenant de l'informatique graphique pour le développement de connaissances architecturales en général, et la classification de formes en particulier. Afin d'expliciter ces tensions, l'étude s'appuie sur un élément porteur de sens à l'échelle du relevé architectural, à savoir la « colonne ». En effet, la colonne reflète et condense les orientations techniques et stylistiques de nombreux courants de l'histoire de l'architecture et c'est pour cette raison qu'elle occupe une place centrale au sein des théories et traités existants depuis la Renaissance (voir les traités de Sebastiano Serlio, Jacopo Barozzi da Vignola, etc.).

\section{De l'intelligibilité par la sémantique}

4 L'intégration des technologies numériques dans l'étude du bâti a pour effet de situer l'objet du relevé hors du lexique architectural. En effet, la pratique du relevé apparait aujourd'hui davantage déconnectée de sa visée culturelle et cognitive dans la mesure où elle tend d'abord vers une démarche centrée sur la récolte de données plus que sur le sens à leur apporter. Bien qu'en partie vraie, cette affirmation mérite néanmoins d'être nuancée.

Une distance s'installe entre la récolte de données sur le terrain et le moment où celles-ci sont analysées et interprétées en laboratoire, ce qui a pour effet d'éloigner le moment d'analyse du travail d'acquisition sur le terrain. Si le relevé peut être défini comme un processus articulant ces deux moments (analyse et acquisition de mesures), il semble que ce dernier soit de plus en plus autonome. Outre une polarisation des activités, c'est la place donnée à la dimension architecturale qui mérite d'être interrogée. Dans ce contexte technologique, une question se pose : qu'est-ce que «le relevé d'architecture » et quelles en sont les spécificités?

6 Dès lors que notre centre d'intérêt porte sur l'objet, dans sa dimension singulière, autonome et matérielle, son relevé suppose de saisir la structure géométrique, l'agencement des formes qui le composent, sa syntaxe et sa grammaire de formes mais aussi et surtout ses logiques sémantiques. Ce dernier aspect pourrait en réalité sortir de la liste tant il regroupe les autres dimensions. Il ne s'agit pas d'envisager l'objet comme un ensemble de termes articulés mais bien de comprendre les relations existant entre la nature géométrique des éléments qui le constituent et leur sens architectural. C'est dans cette tension que s'inscrit la "sémantique architecturale».

7 La caractérisation sémantique constitue sans doute la part la plus tangible de ce qui fonde le relevé d'architecture, non pas parce qu'il s'agit d'un passage obligé (ce n'est pas le cas) 
mais parce que la description sémantique d'un objet exige d'établir une relation entre d'une part un univers géométrique et d'autre part un univers terminologique. Ce type de caractérisation et son expression visuelle sont probablement la meilleure garantie de produire des représentations intelligibles ${ }^{1}$.

Bien qu'il s'agisse d'un des nombreux desseins du relevé d'architecture, l'étude sémantique constitue un fondement de l'analyse architecturale. Un exercice visant à mettre en perspective le « monde des concepts » avec le « monde du réel». Pour évoquer la sémantique d'une forme, Rudolf Arnheim, théoricien de l'art, au xx ${ }^{e}$ siècle ${ }^{2}$, nous donne un exemple singulier : celui de l'hôpital et de la banque. Il se demande comment l'un et l'autre peuvent être identifiés correctement par un usager. Selon lui, « pour comprendre la sémantique architecturale, il serait utile d'examiner le degré de variation possible pour des types de bâtiments donnés. Quelles sont les constantes qui ne peuvent être impunément négligées $\|^{3}$. Nous comprenons ici qu'il s'agit d'appréhender le sens architectural et, par conséquent, l'imaginaire formel, que les termes «hôpital» ou «banque " peuvent véhiculer. La sémantique n'existe qu'à travers la série, la similarité et la régularité d'une relation existant, au sein d'un corpus donné, entre un terme architectural et ce qu'il évoque sur le plan fonctionnel (ou symbolique) et formel. En d'autres termes, nous pouvons parler du «signifié » d'une forme. Le Dictionnaire de l'Académie française définit d'ailleurs le terme « sémantique » comme étant la «science de la signification des mots (...) $»^{4}$.

L'exemple pris par Arnheim porte sur une architecture d'échelle monumentale, mais le raisonnement est facilement transposable au niveau de l'élément. Si l'on considère une colonne comme une entité architecturale à part entière, sa caractérisation sémantique suppose de pouvoir évaluer ce qui la rapproche ou ce qui l'éloigne d'un ensemble d'éléments partageant une fonction semblable (ici une fonction d'abord structurelle), en d'autres mots, d'en évaluer les similarités. Mais sur quoi portent ces similarités ? Partant du principe que la colonne n'existe, ou ne se définit, comme "colonne", qu'à travers d'autres éléments remplissant la même fonction (structurelle, symbolique, etc.), nous comprenons mieux que le relevé et l'analyse d'un objet architectural trouvent notamment du sens à travers la compréhension des éléments " réguliers » ou " typés » qui les composent. Son classement est donc indissociable d'une observation des attributs géométriques convergents ou divergents par rapport à une collection déterminée. En effet, s'il est possible de rassembler sous le "label » sémantique de la « colonne » deux colonnes semblables, cela ne signifie pas pour autant qu'elles le sont en tout point. L'une et l'autre proposent probablement une structure ou sous-structure sémantique différente. Lorsque l'on cherche à évoquer un "type», il s'agit plus d'un terme recouvrant des éléments de «forme» et «fonction» comparables. Au sein d'un même objet, les articulations entre les sous-entités sémantiques sont donc indispensables à la qualification de cet élément.

10 Après la « forme » et la " fonction », considérons à présent la «structure ». En faisant un zoom supplémentaire sur la "colonne», l'«astragale " renvoie formellement à une expression géométrique singulière : le " tore ». Concernant sa «fonction", nous pouvons la considérer de nature esthétique dans la mesure où l'astragale vient assurer la transition entre la verticalité du « fût » et la masse « horizontale » du « chapiteau ». À la lumière de ces deux premières "significations ", surtout la dernière, nous devinons que le «signifié» architectural de l'«astragale» serait altéré si le terme n'était pas implicitement articulé au «fût » et au «chapiteau ». La sémantique de l'« astragale » ne 
peut donc être dissociée des éléments qui l'entourent et qui forment la "colonne». Considérer la sémantique architecturale d'un élément suppose donc de prendre en compte les relations de « voisinage » à travers lesquelles l'élément trouve également son sens. Pour résumer, nous définirions la sémantique d'un élément architectural comme l'expression terminologique d'une relation singulière entre une forme, une fonction et une structure. À partir de cette définition, il est tentant de rapprocher la notion de

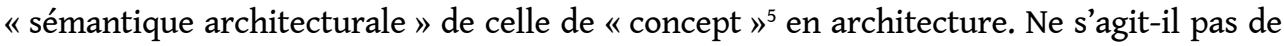
donner une signification architecturale (" sémantisable ») à une forme ou un ensemble de formes par l'utilisation d'instruments de contrôle géométrique comme le dessin?

\section{Sémantique de la colonne romane suivant quatre dictionnaires raisonnés}

11 De manière à mettre en exergue les points de convergence et les points de tension entre une sémantique proposée par l'histoire de l'art et une «sémantique " géométrique issue d'une observation morphologique fine d'éléments semblables formant type, cette étude se focalise sur un corpus d'objets singuliers et homogènes (sur le plan géométrique). Celui-ci est composé de 31 colonnes romanes provenant du cloître de l'abbaye de Saint-Michel de Cuxa (Pyrénées-Orientales), édifié au $\mathrm{XII}^{\mathrm{e}}$ siècle puis en grande partie détruit dans le courant du XIX ${ }^{\mathrm{e}}$. Bien que l'authenticité historique de l'ensemble soit discutable, en raison des nombreuses anastyloses réalisées, il offre un terrain favorable à un tel croisement sémantique. Mais avant d'avancer sur l'analyse morphologique fine du corpus, répondons à une question essentielle, celle du regard posé par l'histoire de l'art sur cet objet. Ce questionnement a pour but de mettre en discussion la lecture de la colonne romane (à partir de dictionnaires raisonnés) avec les observations du modèle géométrique (ou signature morphologique) du corpus.

12 Au-delà d'une description basée sur un vocabulaire architectural stabilisé, l'étude sémantique permet de considérer la structure géométrique de concepts architecturaux singuliers. D'un côté, la sémantique donne une description géométrique des entités lexicales qui composent l'objet; de l'autre côté, la structure géométrique de l'objet est observée indépendamment de la terminologie. Dès lors, deux questions se posent : 1Existe-t-il une convergence géométrique entre la description sémantique (issue de ces dictionnaires) et la description morphologique (provenant de la signature du corpus) ? 2Les traités ou vocabulaires raisonnés d'architecture décrivent-ils de manière uniforme la structure géométrique d'une même entité lexicale ? La réponse à cette seconde question constitue un préalable nécessaire pour répondre à la première. Pour cela, tentons de comprendre si les dictionnaires raisonnés d'architecture décrivent la colonne romane à partir d'une sémantique architecturale stabilisée, en d'autres mots, une sémantique qui renvoie à une structure géométrique et lexicale univoque de l'objet.

13 Sur base de la seule observation visuelle du corpus, toutes les colonnes proposent une décomposition identique. Les discontinuités semblent toutes se trouver aux mêmes endroits. Dès lors, si l'on ne prend que l'une d'entre elles, la S05 (sud 05) par exemple (fig. 1), et que l'on tente de la décrire à partir de différents précis et vocabulaires d'architecture, qu'observe-t-on? Notons que la description proposée (voir fig. 1) ne s'appuie que sur une sélection de dictionnaires étalés dans le temps et faisant autorité (notamment en matière d'architecture médiévale) : Architecture - méthode et vocabulaire ${ }^{6} \mathrm{de}$ 
Pérouse de Montclos, (1972), Précis d'archéologie du Moyen Âge ${ }^{7}$ de Brutails, (1908), Dictionnaire raisonné de l'architecture française du XI ${ }^{e}$ au XVI siècle $e^{8}$ (1858) de Viollet-le-Duc et enfin Des principes de l'architecture, de la sculpture, de la peinture, et des autres arts qui en dépendent : avec un Dictionnaire des termes propres à chacun de ces $\operatorname{arts}^{9}$ de Félibien, (1676).

Figure 1

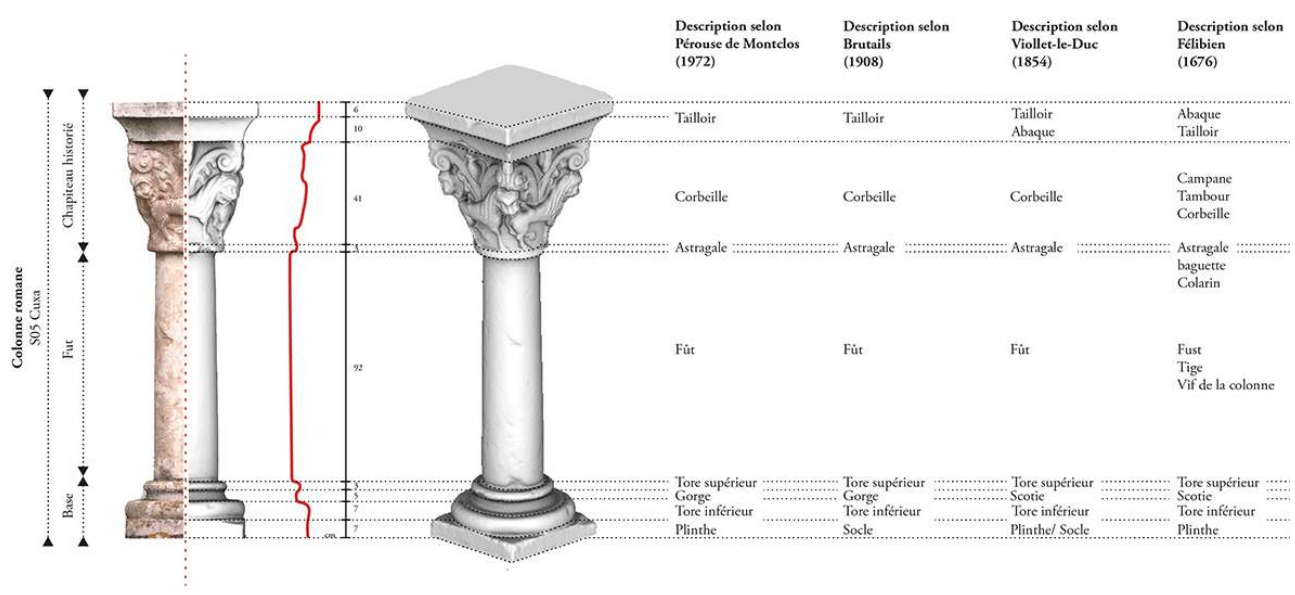

Colonne Sud n5 (S5). Décomposition sémantique suivant les vocabulaires de Pérouse de Montclos (1972), Viollet-le-Duc (1908), Brutails (1854) et Félibien (1676).

(C) David Lo Buglio, Faculté d'architecture, Université libre de Bruxelles (ULB) / UMR 3495 CNRS/MCC MAP.

Comme le montre Jean-Marie Pérouse de Montclos, une colonne romane avec chapiteau historié est structurée autour de concepts architecturaux bien définis. Dans le cas particulier des colonnes de Cuxa, on perçoit clairement chaque passage d'un concept à l'autre. Si ces discontinuités apparaissent distinctement, l'examen de la colonne (S05) à la lumière des quatre dictionnaires nous indique une stabilité relative (dans le temps) des concepts énoncés (voir fig. 1). Ceux-ci s'articulent autour de trois grands ensembles sémantiques («base », « fût », « chapiteau ») et sont décrits comme suit.

\section{La base}

Elle présente un premier corps qui constitue «une assise inférieure carrée, (...) non moulurée ${ }^{10}$. Cette description de la "plinthe » par Pérouse de Montclos trouve une résonance dans la définition du «socle » donnée par Brutails; en témoigne cet extrait : « La base circulaire est posée sur un socle carré, dont les angles sont souvent reliés à la base par des griffes ${ }^{11}$.» On perçoit chez Brutails une inversion entre les concepts de « socle » et de « plinthe » que cet autre extrait vient confirmer : « La base est posée sur un socle qui, (...) est lui-même monté sur une plinthe couronnée d'une moulure ${ }^{12}$.» Pour lui, la première partie moulurée se trouve donc posée sur un socle et non sur une plinthe. En revanche, si Félibien et Viollet-le-Duc parlent de "plinthe" pour décrire ce premier élément de la colonne romane, Viollet-le-Duc parle également de « socle » lorsqu'il définit le premier lit carré de la base attique ${ }^{13}$. 
16 En poursuivant l'examen de la base, on remarque que le «tore inférieur » et le «tore supérieur» renvoient dans les quatre ouvrages à «une moulure pleine de profil curviligne $»^{14}$. La seule différence est d'ordre terminologique et concerne la «scotie », le profil en creux situé entre les deux tores. Parce qu'elle évoque davantage le langage classique, la "scotie » est remplacée au xxe siècle, par Brutails et Pérouse de Montclos, par la «gorge ». En parlant de la base attique, ce dernier nous indique d'ailleurs que «dans l'architecture médiévale, une gorge de profil quelconque remplace la scotie ${ }^{15}$.

\section{Le fût}

17 Cette partie centrale de la colonne fait la liaison entre le socle et le chapiteau. "C'est habituellement un cylindre. Il n'est donc nécessaire de le préciser que pour opposer la colonne cylindrique à la colonne ovale ou polygonale ${ }^{16}$. » Si le terme « fût » est commun aux trois dictionnaires les plus récents, les indications données par Brutails quant à la nature géométrique de l'élément sont plus précises que celles proposées par Viollet-leDuc. Alors que pour le premier, "les fûts [de la colonne romane] sont cylindriques, rarement renflés, quelquefois peints ou semés de menus ornements sculptés en faible relief $»^{17}$, le second se contente de les décrire comme une " partie de la colonne comprise entre la base et le chapiteau ${ }^{18}$. Précisons que dans le cas spécifique des colonnes du cloître de Cuxa, il s'agit de cylindres qui laissent, dans quelques cas, deviner une légère augmentation de la section en pied de « fût ». Le quatrième vocabulaire, celui de Félibien, parle $\mathrm{du}$ « fust » de la colonne comme du « corps de la colonne compris entre la base \& le chapiteau $\aleph^{19}$. Bien que cette description lapidaire se rapproche de celle donnée par Viollet-le-Duc, on remarque qu'il ne s'agit pas dans son vocabulaire d'un terme stabilisé. Il a fréquemment recours à des synonymes pour exprimer le même élément sémantique tels que le « vif de la colonne $»^{20}$ ou encore la « tige » (du latin « scapus ») qu'il reprend, selon ses termes, de Vitruve ${ }^{21}$.

\section{Le chapiteau}

La première discontinuité, marquant la transition entre le groupe sémantique médian et le groupe supérieur de la colonne, signale le début de l'« astragale ». Pérouse de Montclos le décrit comme un « corps de moulures séparant le chapiteau du fût (...) généralement une baguette $»^{22}$, qu'il spécifie comme étant une "moulure pleine dont la largeur est sensiblement égale à la saillie et le profil, un segment de cercle $»^{23}$. Si pour Brutails l'« astragale » se "réduit à la forme d'un simple tore " ${ }^{24}$, Viollet-le-Duc en donne une définition plus approfondie : "quelquefois, à cette époque de recherche dans l'exécution des profils [aux alentours du XII ${ }^{\mathrm{e}}$ siècle], le tore de l'astragale, au lieu de présenter en coupe un demi-cercle, est aplati $»^{25}$, ce qui semble correspondre au profil de l'« astragale » de la colonne S05. Malgré les quelques nuances ou compléments apportés par les trois dictionnaires les plus récents, la description sémantique qu'ils donnent de l'élément reste stable. À l'inverse, l'ouvrage de Félibien parle aussi de «baguette $»^{26}$ ou plus encore de " colarin " pour décrire « le haut du vif de la colonne »"27.

Peut-être moins évidente, la discontinuité suivante marque la transition entre l'« astragale » et la « corbeille » du chapiteau. Alors que Félibien évoque la « corbeille » à travers différents synonymes, le "tambour ", et plus particulièrement la "campane $»^{28}$ (en référence à la cloche renversée), les autres vocabulaires s'accordent autant sur la 
terminologie de l'élément que sur sa description géométrique. Pour ne retenir que la plus évocatrice, Brutails définit la " corbeille » romane comme une "transition [géométrique] entre le fût circulaire et plus petit, d'une part, et le tailloir carré et plus large, de l'autre » 29 .

Enfin, si la discontinuité suivante indique le passage vers le "tailloir", celle d'après marque une nouvelle transition géométrique ne renvoyant à aucune variation terminologique. De toutes les discontinuités apparentes, une seule n'articule pas deux concepts mais se retrouve englobée dans un seul et même élément sémantique, le «tailloir " (voir fig. 1). Dans le cas de la colonne S05, le "tailloir " comprend donc un parallélépipède chanfreiné (dont le profil s'approche du "cavet renversé $»^{30}$ ), surmonté d'un parallélépipède rectangle. Si la description donnée par Pérouse de Montclos est l'une des plus satisfaisantes, elle indique peu de choses sur le plan géométrique. Il s'agit pour lui $\mathrm{du}$ « couronnement du corps de certains chapiteaux, formé par une assise ou ayant sensiblement la hauteur d'une assise $»^{31}$. Au regard des quatre dictionnaires d'architecture, le «tailloir » apparait assez flou et laisse un champ de questionnements ouverts sur la nature géométrique de cette entité sémantique. Concernant les aspects terminologiques, le mot ne se stabilise qu'au $\mathrm{xx}^{\mathrm{e}}$ siècle dans les dictionnaires de Pérouse de Montclos et Brutails. Avant cela, l'utilisation du terme "abaque » est courante chez Viollet-le-Duc et Félibien, voire plus importante. C'est Brutails qui, le premier des quatre, emploie le mot «tailloir » de manière systématique pour parler du couronnement de la colonne romane. Il considère en effet le «tailloir» comme une «réminiscence de l'« abaque » romain $»^{32}$.

21 Ces observations nous racontent deux choses. La première concerne la variabilité dans le temps de la sémantique architecturale (sur les trois derniers siècles). Si l'on s'aperçoit qu'aux $\mathrm{XIX}^{\mathrm{e}}$ et $\mathrm{xx}^{\mathrm{e}}$ siècle la description terminologique des éléments se stabilise, elle est encore "changeante» avant cette période (du moins pour la colonne romane). La seconde chose à retenir est l'interdépendance qui existe entre la structuration terminologique d'une colonne et sa structuration géométrique. On remarque que les discontinuités observables articulent des concepts architecturaux bien déterminés. Toutefois, il subsiste quelques écarts d'interprétation quant à la description géométrique de certains éléments.

\section{Sémantique du modèle théorique vs sémantique du modèle géométrique de Cuxa}

Afin d'affiner l'examen sémantique réalisé sur la colonne romane et d'identifier l'apport de la complexité des représentations numériques dans l'étude du bâti, ce point s'intéresse à la création d'un modèle géométrique. En s'appuyant sur le principe d'auto-extension d'un ensemble de données, l'approche vise à identifier automatiquement une structure géométrique commune à une collection d'artefacts, soit une signature morphologique, fruit d'un travail d'analyse morphologique et statistique réalisé sur les éléments numérisés. Elle caractérise le corpus, du moins sur un jeu déterminé d'attributs géométriques.

Tout comme la seule empreinte digitale ne suffit pas à cerner la personnalité d'un individu, la signature morphologique ne révèle pas la nature stylistique profonde de la collection mais en donne une empreinte unique et distinctive. Cette signature ne peut 
donc être considérée comme la représentation graphique d'un artefact numérisé ni même comme un "modèle théorique ». Si la création d'une signature ne s'apparente pas à une méthode d'analyse à proprement parler, elle propose cependant un regard synthétique sur la collection et conduit l'analyste à en observer plus finement ses caractères singuliers.

Figure 2

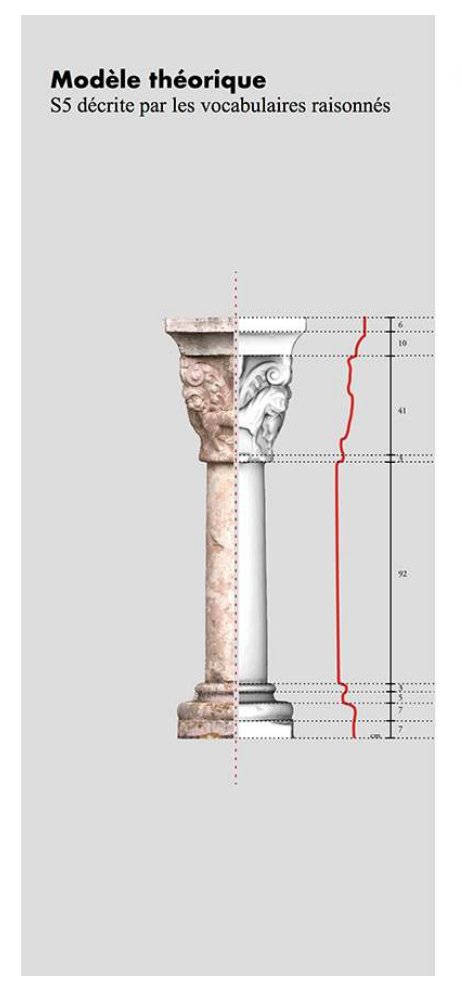

\section{Signature de discontinuités}

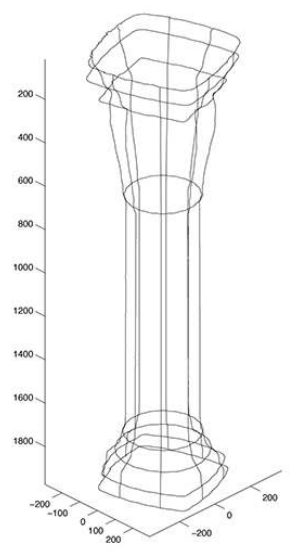

Confrontation entre le modèle théorique (issu de l'observation de quatre dictionnaires raisonnés) et la signature de discontinuités des colonnes du cloître de l'abbaye de Saint-Michel-de-Cuxa.

(c) David Lo Buglio, Faculté d'architecture, Université libre de Bruxelles (ULB) / UMR 3495 CNRS/MCC MAP. 


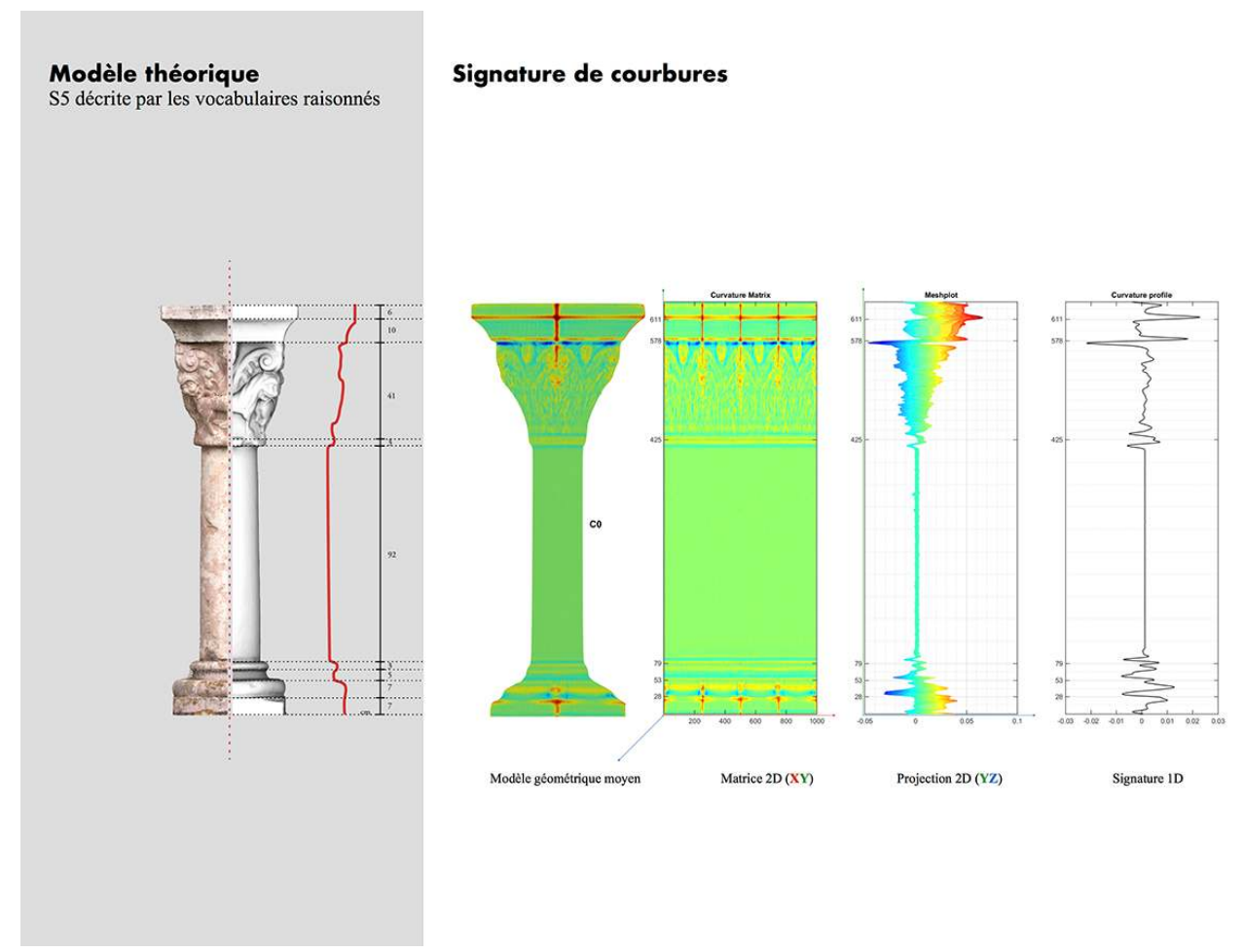

Confrontation entre le modèle théorique (issu de l'observation de quatre dictionnaires raisonnés) et la signature de courbures des colonnes du cloître de l'abbaye de Saint-Michel-de-Cuxa.

(c) David Lo Buglio, Faculté d'architecture, Université libre de Bruxelles (ULB) / UMR 3495 CNRS/MCC MAP.

Dans le cas spécifique de cette étude, la signature du corpus exprime deux caractéristiques de chaque entité : les courbures de surface et les discontinuités de forme (fig. 2, fig. 3). En effet, la plupart des formes peuvent être décrites par les courbures de surface et les discontinuités qui les composent. L'étude des discontinuités permet d'appréhender automatiquement les différentes articulations sémantiques présentes dans la colonne. L'expression "discontinuités de forme» signifie que chaque changement important de la surface est marqué par une variation clairement perceptible ; en d'autres termes, lorsque la variation (angulation) dépasse un facteur « $\mathrm{n}$ » de déformation. Dans le cas particulier de l'étude d'éléments architecturaux, les discontinuités de forme fournissent une compréhension fine des articulations géométriques (voire sémantiques) de l'objet. De manière plus triviale, il s'agit des limites et divisions observables. Comme indiqué précédemment, une forme peut également être décrite à partir de ses courbures de surface. Ces dernières correspondent à l'expression mathématique des variations d'une surface en chacun de ses points. Sans rentrer dans des considérations trop techniques, l'étude des courbures permet une approche mathématique efficace pour la caractérisation géométrique de n'importe quelle surface.

Le croisement des deux attributs géométriques sur l'ensemble du corpus a permis d'obtenir une signature qui, à défaut d'offrir un véritable "modèle théorique ", fournit un nouveau prisme de lecture de la colonne romane de Cuxa (voir fig. 2, fig. 3). Nous nous focalisons ici sur les enjeux sémantiques, et, pour plus d'informations sur les aspects 
techniques de l'étude (et l'implémentation des signatures), nous renvoyons le lecteur à l'article : «What do 31 columns tell about a "theoretical" 32nd? » ${ }^{33}$.

Regardons à présent l'apport des signatures à la caractérisation sémantique. La description donnée ici de notre colonne romane s'appuie sur quatre vocabulaires raisonnés d'architecture. Il n'est évidemment pas question de discuter l'objet théorique à partir d'un corpus unique mais plutôt de comprendre s'il est possible de situer les tensions existant entre une sémantique proposée par l'histoire de l'art et la signature issue du travail de caractérisation morphologique.

Pour ne retenir que les observations les plus intéressantes : au niveau de la «base », si la signature de courbures montre une articulation de quatre éléments, à savoir la "plinthe ", le "tore inférieur", la "gorge » et le "tore supérieur", la signature de discontinuités fait en revanche disparaître un élément. Les discontinuités détectées indiquent une transition entre la "plinthe » et le "tore inférieur", puis entre le "tore inférieur » et le « tore supérieur » sans que la " gorge » ne soit identifiée. Cette curiosité peut en partie s'expliquer par la faible concavité des « gorges ». En théorie, ces dernières correspondent à un profil en demi-cercle qui, articulé au profil inverse du "tore", marque une rupture franche. Ce n'est pas le cas ici. La signature du corpus montre que la majorité des profils des «gorges» ne forment pas un demi-cercle mais sont plus aplatis. Toutefois, comme l'indique Brutails en parlant de la colonne romane, cette caractéristique ne semble pas propre à notre corpus : «la colonne a toujours une base, laquelle transmet au socle la pesée que reçoit le fût. Cette base est habituellement la base attique; mais la scotie peut être remplacée par une gorge plus ou moins haute, plus ou moins sèche. Ces profils varient, d'ailleurs, d'après l'angle sous lequel ils doivent être vus et d'après la dureté de la pierre ${ }^{34}$. »

Dans le cas du "fût», la signature de discontinuités marque convenablement sa transition avec les autres parties de la colonne, tandis que la signature de courbures donne des résultats proches de 0 , c'est-à-dire une aire minimale, comme celle d'un cylindre. Au niveau de l'« astragale », les signatures révèlent deux choses intéressantes et quasiment contradictoires. La première, la signature de courbures, identifie des variations de surface distinctes de celles des courbures propres au «fût " ou à la "corbeille». Par contre, la signature de discontinuités ne parvient pas à isoler correctement l'«astragale » (fig. 4). Des deux discontinuités devant circonscrire l'élément, seule la discontinuité inférieure apparait. En termes de structure géométrique, la signature associe ici l'« astragale » avec la « corbeille » du « chapiteau ». La signature vérifie ainsi les propos de l'historien Brutails qui explique qu'« à l'exception de quelques provinces restées fidèles à l'antiquité classique, l'astragale, réduit à un simple tore, fait corps avec le chapiteau et non plus avec le fût ». La signature vient non seulement confirmer le propos de Brutails mais contribue également à le rendre explicite. 


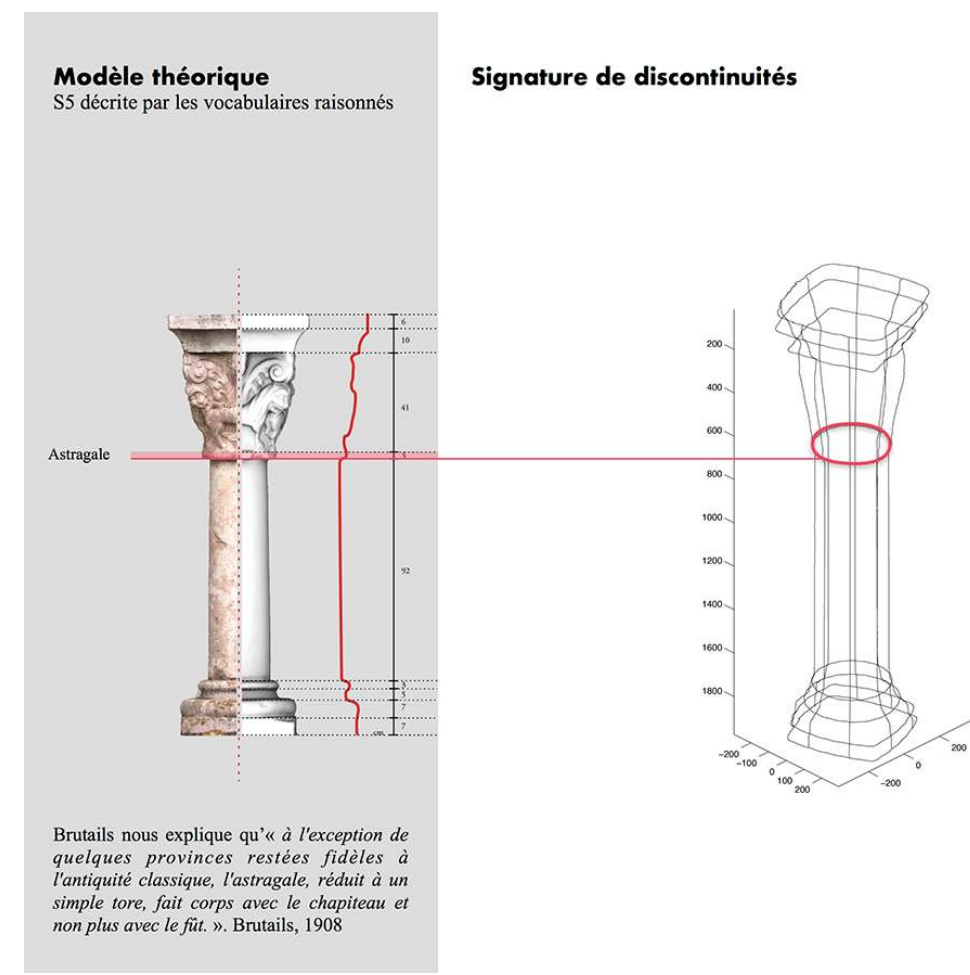

Confrontation entre le modèle théorique (issu de l'observation de quatre dictionnaires raisonnés) et la signature morphologique des colonnes du cloitre de l'abbaye de Saint-Michel-de-Cuxa. - observation de l'astragale.

(c) David Lo Buglio, Faculté d'architecture, Université libre de Bruxelles (ULB) / UMR 3495 CNRS/MCC MAP.

29 Si plusieurs considérations ont déjà été fournies pour la «corbeille ", les «tailloirs » méritent qu'on s'y attarde un moment. Tant la signature de discontinuités que la signature de courbures indique une rupture nette au milieu de l'entité sémantique. Pourtant, aucun des vocabulaires raisonnés ne fait état d'une structure géométrique aussi singulière (fig. 5). L'incohérence existant entre l'observation morphologique et la description sémantique est révélatrice de l'ambiguïté de ce terme. Il s'agit d'une entité peu ou mal définie dont la distinction avec l'« abaque » apparaît plus ou moins clairement suivant les auteurs. Sur la base de l'analyse morphologique, on pourrait presque parler du bas et du haut tailloir. 


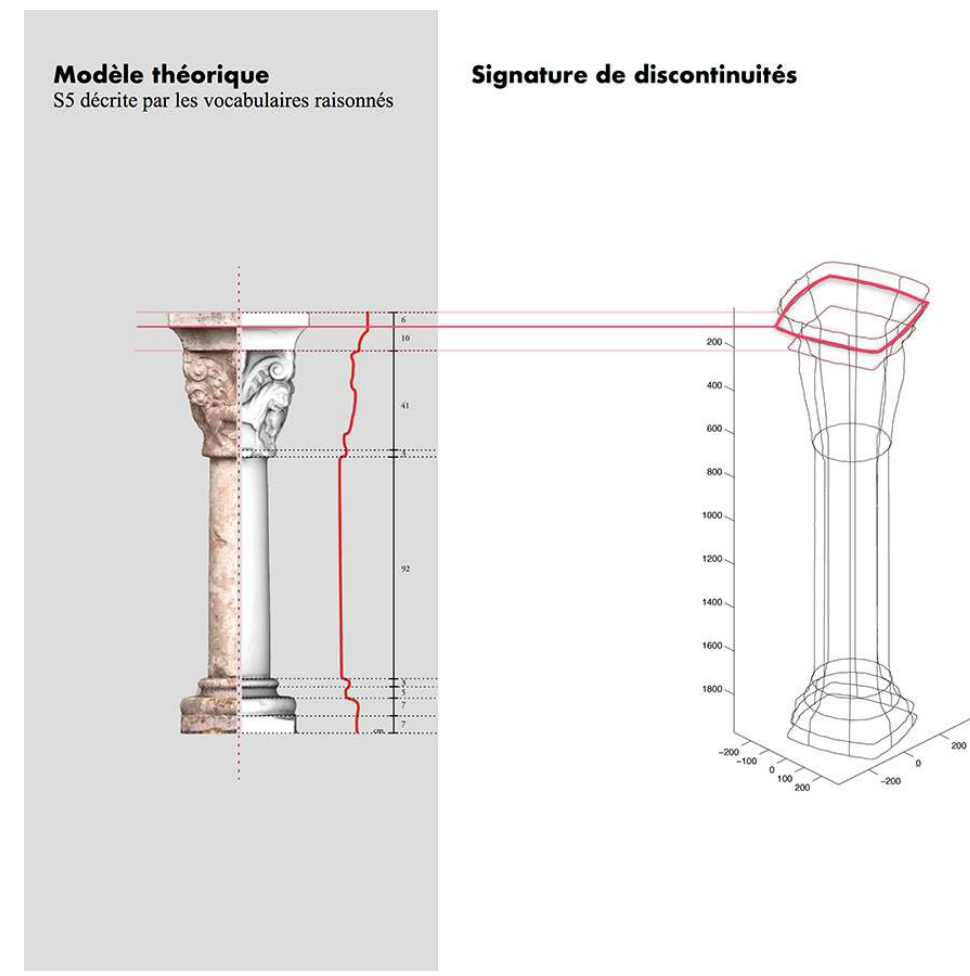

Confrontation entre le modèle théorique (issu de l'observation de quatre dictionnaires raisonnés) et la signature morphologique des colonnes du cloître de l'abbaye de Saint-Michel-de-Cuxa - observation du tailloir.

(c) David Lo Buglio, Faculté d'architecture, Université libre de Bruxelles (ULB) / UMR 3495 CNRS/MCC MAP.

\section{Conclusion}

Dépourvues du regard de l'observateur, ces signatures n'ont aucune valeur intrinsèque. $\mathrm{Ni}$ artefacts, ni «modèle théorique", leur intérêt se situe davantage dans les questionnements qu'elles ouvrent. L'exploitation des numérisations et la formalisation de signatures morphologiques offrent de nouveaux moyens pour la classification d'éléments architecturaux. Si aujourd'hui l'étude s'appuie sur un corpus limité à une dizaine de colonnes, qu'en serait-il si elle était étendue à 300 ou 3000 ? Et en corollaire, à la lumière de cette approche, n'est-il pas tentant de discuter ou de rediscuter les définitions des vocabulaires?

31 En croisant la caractérisation géométrique du corpus avec la description fournie par les vocabulaires d'architecture, il s'agit d'explorer une nouvelle méthode d'assistance à l'analyse. L'approche défendue ici se justifie d'une part par le contexte actuel de démocratisation des outils de collecte de données et d'autre part par le besoin de méthodologies innovantes pour l'exploitation de données massives. Ce travail fournit ainsi une première "boîte à outils " pour l'assistance à la caractérisation sémantique d'un jeu de formes singulier. L'ouverture de ce champ de recherche offre également des opportunités pour 1) l'étude des altérations morphologiques, 2) l'indexation et la classification d'éléments architecturaux, 3) l'étude des propagations stylistiques dans le temps et dans l'espace. 
Le recours aux outils d'analyse de formes provenant d'autres champs disciplinaires démontre que le cumul de données peut soutenir une certaine forme d'intelligibilité des représentations numériques en renouvelant notre regard sur la sémantique architecturale. À bien des égards, la " particularité » s'avère davantage éclairante sur la série que la "norme» elle-même. Ce travail d'analyse "retour» et le caractère surprenant de certaines observations ne peuvent aboutir sans l'existence de conditions favorables, celles établies par la création de signatures morphologiques. Cette démarche ouvre ainsi la voie à d'autres formes d'intelligibilité. Nous pouvons, en ce sens, peut-être parler d'une intelligibilité de bas niveau, d'une intelligibilité de l'indice, de la trace, de l'anomalie.

\section{NOTES}

1. - Dans le cadre de cette étude, la notion d'intelligibilité exprime la capacité de la numérisation (de la représentation 3D ou de la représentation de manière plus générale) à renvoyer à un univers de connaissances propres au domaine architectural, en somme un univers susceptible de nous informer sur la sémantique de l'objet.

2. - SORENSEN, Lee. «Arnheim, Rudolf». Dans Dictionary of Art Historians. Durham (Caroline du Nord): Duke University, 2016. Voir les sites: https://dictionaryofarthistorians.org/index.htm. http://arthistorians.info/arnheimr [consulté le 10/04/2019].

3. - ARNHEIM, Rudolf. Dynamique de la forme architecturale [1977]. Bruxelles/Liège : Mardaga, 1986, p. 206.

4. - Académie française. Dictionnaire de l'Académie française. $8^{e}$ éd. Paris : Hachette, 1935, 2. H-Z. http://www.cnrtl.fr/definition/academie8/sémantique [consulté le 10/04/2019].

5. - Le Dictionnaire de l'Académie, dans sa neuvième édition (1985), propose la définition suivante: «En logique: construction de l'esprit explicitant un ensemble stable de caractères communs désigné par un signe verbal (...) En épistémologie: en mathématiques, notion rigoureusement définie qui sert de fondement ou de principe. Le concept de cercle, de triangle, de nombre, d'ensemble, de sous-ensemble. ». Référence, voir glossaire.

6. - PÉROUSE de MONTCLOS, Jean-Marie. Architecture - Méthode et vocabulaire [1972]. Paris : Éd. du Patrimoine, 2000 ( $3^{\mathrm{e}}$ éd.).

7. - BRUTAILS, Jean-Auguste. Précis d'archéologie du Moyen Âge. Toulouse/Paris : Édouard Privat/ Picard, 1908.

8. - VIOLLET-LE-DUC, Eugène Emmanuel. Dictionnaire raisonné de l'architecture française du XI au XVI ${ }^{e}$ siècle. Paris : B. Bance, 1854.

9. - FÉLIBIEN, André. Des principes de l'architecture, de la sculpture, de la peinture, et des autres arts qui en dépendent : avec un dictionnaire des termes propres à chacun de ces arts. Paris : chez Jean-Baptiste Coignard, 1676. Voir le site: http://gallica.bnf.fr/ark:/12148/bpt6k50597k [consulté le 10/04/2019].

10. - PÉROUSE de MONTCLOS, Jean-Marie. Architecture - Méthode et vocabulaire. Op. cit., p. 230.

11. - BRUTAILS, J.-A. Op. cit., p. 92.

12. - Ibid., p. 176.

13. - VIOLLET-LE-DUC, E. E. Op. cit., vol. II, p. 132.

14. - PÉROUSE de MONTCLOS, J.-M. Op. cit., p. 365. 


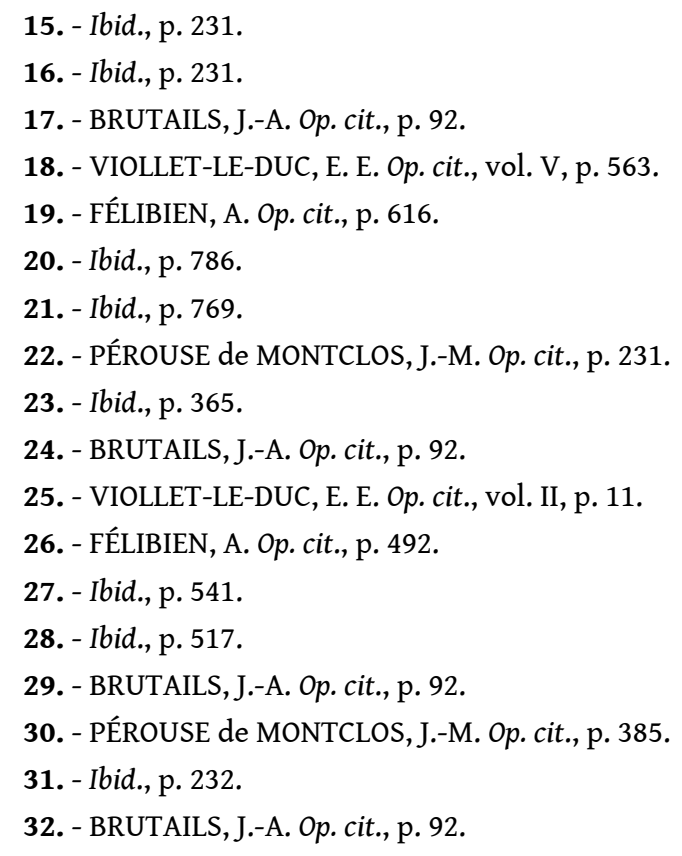

33. - LO BUGLIO, David, LARDINOIS, Vanessa, et DE LUCA, Livio. « What do 31 columns say about a "theoretical" 32nd?». ACM Journal on Computing and Cultural Heritage, 2015, 8, $\mathrm{n}^{\circ} 1$. Voir le site : https://doi.org/10.1145/2700425 [consulté le 10/04/2019].

34. - BRUTAILS, J.-A. Op. cit., p. 92.

\section{RÉSUMÉS}

En articulant terminologie, logique fonctionnelle et logique géométrique, la sémantique d'un élément peut être considérée comme un concept d'architecture à part entière. Non seulement essentielle à la compréhension de l'évolution stylistique dans le temps et dans l'espace, la sémantique architecturale constitue à bien des égards l'un des fondements des théories d'architecture. En se situant à la croisée de la théorie d'architecture, de l'histoire de l'art, voire des pratiques du relevé, les vocabulaires d'architecture offrent des tentatives de normalisation sémantique qui pourraient être rediscutées à la lumière des avancées scientifiques récentes dans le domaine de l'étude du bâti. Cet article vise donc à concilier les enjeux cognitifs propres à l'étude sémantique d'éléments architecturaux et l'exploitation de la donnée massive issue des représentations numériques. Il s'agit donc de croiser la caractérisation géométrique d'un corpus de formes (résultant de l'examen des similarités géométriques partagées par ce corpus) avec la description donnée par quatre vocabulaires d'architecture. L'enjeu est d'observer les points de rencontre mais aussi de tension entre une sémantique proposée par l'histoire de l'art et une sémantique issue d'une observation géométrique fine. En mettant en exergue les contradictions entre les vocabulaires eux-mêmes et le modèle géométrique obtenu, cet article discute l'apport des méthodes d'analyse provenant de l'informatique graphique dans le développement de connaissances architecturales.

By articulating terminology, functional logics and geometrical logics, the semantics of an element can be considered as an architectural concept. In many ways, architectural semantics is 
one of the foundations of architectural theory, essential for understanding stylistic evolutions in time and space. At the crossroads of architectural theory, art history and even survey practices, architectural vocabularies offer efforts to provide semantic standardisation that might be revisited today, in the light of recent scientific advances in the field of the built heritage. This article aims to reconcile the cognitive issues specific to the semantic study of architectural elements and the exploitation of big data resulting from building digitisations. It is necessary to compare the geometrical characterisation of a corpus of elements (resulting from the examination of the geometrical similarities shared by this corpus) with the description given by four architectural vocabularies. The challenge is to observe the encounters, but also the points of tension between a semantics proposed by the history of art and a semantics resulting from fine geometrical observations. By highlighting the contradictions between the vocabularies themselves and the geometric model, this article discusses the contribution of methods of analysis coming from computer graphics for the development of architectural knowledge.

\section{INDEX}

Keywords : architectural semantics, architecture, documentary record, architectural analysis, (architectural) drawing, representation, form, meaning, Dictionnaire raisonné de l'Architecture, vocabulary, 3D digitisation, digital

Mots-clés : Sémantique architecturale, architecture, relevé, analyse architecturale, dessin (d'architecture), représentation, forme, sens, dictionnaire raisonné de l'Architecture, vocabulaire, numérisation 3D, numérique

\section{AUTEUR}

\section{DAVID LO BUGLIO}

Chargé de cours à la Faculté d'architecture, Université libre de Bruxelles (ULB) - Belgique, Chercheur associé à l'UMR 3495 CNRS/MCC MAP - France david.lo.buglio@ulb.ac.be 